\title{
Improvement in left ventricular function assessed by tissue Doppler imaging after aortic valve replacement for severe aortic stenosis
}

Chih-Chiang Nieh ${ }^{1}$, MBBs, MrCs, Alvin Yeng-Hok $\underline{\text { Teo }}^{1}$, MBBS, Wern Miin Soo ${ }^{1}$, MBBS, MrCP, Glenn K $\underline{\text { Lee }}^{1}$, MBBS, Devinder Singh ${ }^{1}$, MBBS, MRCP, Kian-Keong $\underline{P o h}^{1,2}$, FRCP, FACC

INTRODUCTION The effects of reduction of left ventricular (LV) systemic afterload following aortic valve replacement (AVR) for severe aortic valve stenosis (AS) were investigated, using echocardiography and tissue Doppler imaging (TDI). METHODS We compared the preoperative and postoperative echocardiographic assessments of 23 patients with severe AS who had undergone isolated AVR $(n=13)$ or concomitant AVR with coronary artery bypass grafting $(C A B G)(n=10)$. Conventional echocardiographic evaluations and TDI at the lateral mitral annulus were performed.

RESULTS Echocardiography was performed at a median of 120 (interquartile range: 66-141) days after AVR. There was significant reduction in aortic transvalvular mean pressure gradient after AVR. Although LV dimensions, mass and ejection fraction remained unchanged, LV diastolic and systolic functions improved (as observed on TDI). Early diastolic (E'), late diastolic (A') and systolic (S') mitral annular velocities increased significantly $(p<0.05)$. There was significant improvement in TDI-derived parameters among the patients who had isolated AVR, while among the patients who had concomitant AVR with CABG, only S' had significant improvement $(p=0.028)$.

CONCLUSION TDI was able to detect improvements in LV systolic and diastolic function after AVR for severe AS. There was less improvement in the TDI-derived diastolic parameters among patients who underwent concomitant AVR with CABG than among patients who underwent isolated AVR.

Keywords: aortic valve replacement, aortic valve stenosis, coronary artery bypass grafting, left ventricular function, tissue Doppler imaging

\section{INTRODUCTION}

Aortic valve stenosis (AS) is the most common valvular disease in developed countries; it is also the third most common cardiovascular pathology after coronary artery disease (CAD) and hypertension. (1) The natural history of AS is defined by a long asymptomatic phase that may persist for several decades. Progressive left ventricular (LV) dysfunction may result from prolonged LV outflow obstruction. ${ }^{(2)}$ Aortic valve replacement (AVR) is the definitive treatment for severe AS in patients who are symptomatic, who have decreased LV ejection fraction, or who are scheduled to undergo concomitant cardiac surgery..$^{(3,4)}$ As AS and CAD may have similar risk factors, and are both known to have increased incidence and disease progression with increasing age, they often coexist in elderly patients. Patients with severe AS and CAD who are undergoing surgery have significantly higher surgical risk as compared to those with isolated severe AS. However, in the last few decades, the number of patients undergoing concurrent AVR with coronary artery bypass grafting (CABG) has doubled. ${ }^{(5)}$

Tissue Doppler imaging (TDI) is a useful echocardiographic technique that can be used to assess myocardial tissue velocity and detect subclinical LV systolic and diastolic dysfunctions. ${ }^{(6)}$ In the present study, we aimed to investigate the effects of reduction in systemic afterload on the left ventricle after AVR in severe AS. We compared (a) the preoperative and postoperative echocardiographic parameters, including those measured using TDI; and (b) the subsequent improvements in LV function between patients who underwent isolated AVR and those who underwent concomitant AVR and CABG.

\section{METHODS}

A total of 23 patients (14 male, nine female) who underwent AVR for severe AS and had paired echocardiographic studies, including TDI, at the National University Heart Centre, Singapore, were included in our study. Among these 23 patients, 13 underwent isolated AVR, while ten underwent concomitant AVR and CABG. Their mean age was $65 \pm 8$ years. Patients with atrial fibrillation, pacing devices and significant mitral valve regurgitation were excluded.

All 23 patients were assessed comprehensively on transthoracic echocardiography, before and after AVR. The images were stored on a dedicated workstation for offline analysis. The average of three cardiac cycles was taken for each measurement. Continuous wave Doppler ultrasonography was used to measure aortic transvalvular peak velocity. The mean pressure gradient was calculated from the tracing of the velocity curve. Aortic valve area was derived using the continuity equation. ${ }^{(7)}$ LV enddiastolic and end-systolic volumes and LV ejection fraction were measured according to the American Society of Echocardiography guidelines. ${ }^{(8)}$ LV diastolic function was assessed using transmitral

\footnotetext{
${ }^{1}$ Department of Cardiology, National University Heart Centre Singapore, National University Health System, ${ }^{2}$ Department of Medicine, Yong Loo Lin School of Medicine, National University of Singapore, Singapore

Correspondence: A/Prof Kian-Keong Poh, Senior Consultant and Associate Professor, Department of Cardiology, National University Heart Centre, 1 E Kent Ridge Road, NUHS Tower Block, Level 9, Singapore 119228. kian_keong_poh@nuhs.edu.sg
} 
Table I. Demographics of patients who underwent isolated aortic valve replacement (AVR) or AVR with coronary artery bypass grafting (CABG) $(n=23)$.

\begin{tabular}{|c|c|c|c|}
\hline \multirow[t]{2}{*}{ Variable } & \multicolumn{2}{|c|}{ Mean \pm standard deviation } & \multirow[t]{2}{*}{ p-value } \\
\hline & Isolated AVR $(\mathrm{n}=13)$ & CABG + AVR $(n=10)$ & \\
\hline \multicolumn{4}{|l|}{ Gender* } \\
\hline Male & $9(69.2)$ & $5(50.0)$ & NS \\
\hline Female & $4(30.8)$ & $5(50.0)$ & NS \\
\hline $\mathrm{Age}^{+}(\mathrm{yr})$ & $63.0(57-69)$ & $69.5(64-77)$ & NS \\
\hline Body mass index $\left(\mathrm{kg} / \mathrm{m}^{2}\right)$ & $24.6 \pm 4.2$ & $24.8 \pm 4.5$ & NS \\
\hline Duration from surgery to postoperative echocardiography ${ }^{+}$(day) & $101(53-138)$ & $125(110-146)$ & NS \\
\hline LV end-diastolic volume (mL) & $110 \pm 45$ & $113 \pm 11$ & NS \\
\hline LV end-systolic volume (mL) & $42 \pm 36$ & $49 \pm 29$ & NS \\
\hline LV ejection fraction (\%) & $62 \pm 14$ & $61 \pm 5$ & NS \\
\hline LV mass index $\left(\mathrm{g} / \mathrm{m}^{2}\right)$ & $118 \pm 50$ & $120 \pm 23$ & NS \\
\hline Aortic transvalvular peak velocity $(\mathrm{cm} / \mathrm{s})$ & $259 \pm 90$ & $254 \pm 91$ & NS \\
\hline Aortic transvalvular mean gradient $(\mathrm{mmHg})$ & $40 \pm 15$ & $43 \pm 18$ & NS \\
\hline Aortic valve area $\left(\mathrm{cm}^{2}\right)$ & $0.85 \pm 0.41$ & $0.79 \pm 0.30$ & NS \\
\hline Aortic valve resistance (dyne.s/cm ${ }^{5}$ ) & $240 \pm 111$ & $240 \pm 206$ & NS \\
\hline$E^{\prime}(\mathrm{cm} / \mathrm{s})$ & $8.27 \pm 2.47$ & $7.69 \pm 2.47$ & NS \\
\hline$A^{\prime}(\mathrm{cm} / \mathrm{s})$ & $12.02 \pm 3.62$ & $9.54 \pm 2.49$ & NS \\
\hline$S^{\prime}(\mathrm{cm} / \mathrm{s})$ & $8.79 \pm 1.63$ & $7.23 \pm 1.51$ & 0.027 \\
\hline
\end{tabular}

*Data presented as no. (\%). †Data presented as median (interquartile range). A': late diastolic mitral annular velocity; E': early diastolic mitral annular velocity; LV: left ventricular; NS: not significant; S': systolic mitral annular velocity

early (E) and late (A) filling velocities, and deceleration time was obtained from pulse wave Doppler interrogation at the mitral leaflet tips. Pulse wave TDI was performed in apical fourchamber view to assess longitudinal myocardial velocity. ${ }^{(9)}$ Peak velocities during systole $\left(\mathrm{S}^{\prime}\right)$, and early $\left(\mathrm{E}^{\prime}\right)$ and late $\left(\mathrm{A}^{\prime}\right)$ diastole at the lateral mitral annulus were measured, and $E / E^{\prime}$ ratio was calculated. Conventionally, TDI parameters are obtained over both the septal and lateral mitral annulus and the average of the values is used. However, TDI parameters at the septal annulus were excluded in the present study due to the known effect of CABG on these readings.

Data collection and statistical analysis were performed using Microsoft Excel for Windows 2010 (Microsoft, Redmond, WA, USA). Statistical tests of significance were performed and a $p$-value $<0.05$ indicated statistical significance. Continuous variables were presented as mean \pm standard deviation or median (interquartile range $[\mathrm{IQR}]$ ), depending on the normality of the distribution, and analysed using the two-tailed Student's $t$-test (for variables with continuous distributions) or Mann-Whitney $U$ test (for variables with unknown distributions). Categorical or dichotomous data was presented as number (percentage) and analysed using chi-square tests.

\section{RESULTS}

The demographics of the 23 patients, according to whether they underwent isolated AVR $(n=13)$ or AVR with CABG $(n=10)$, are listed in Table I. Transthoracic echocardiography was performed at a median of 120 (IQR 66-141) days after AVR.

After AVR, there was a significant increase in aortic valve area $(p<0.001)$ and a significant decrease in aortic transvalvular mean pressure gradient $(p<0.001)$ (Table II). In addition, aortic transvalvular peak velocity $(\mathrm{p}<0.001)$ and aortic valve resistance $(p=0.006)$ decreased significantly after AVR. Although LV dimensions, mass and ejection fraction remained unchanged after AVR, there was improvement in LV diastolic and systolic function, as observed on TDI. Among the patients who underwent isolated AVR $(n=13)$, all TDI parameters improved (Table II \& Fig. 1). Among the patients who underwent AVR with CABG $(n=10)$, there was only significant improvement in $S^{\prime}(p=0.028)$; although there was an increasing trend in $\mathrm{E}^{\prime}$ and $\mathrm{A}^{\prime}$, the changes in these velocities were not statistically significant (Table II). Interestingly, a comparison of the preoperative baseline TDI parameters of the patients who underwent isolated AVR and those who underwent AVR with CABG showed that $S^{\prime}$ was significantly higher in the isolated AVR group $(p=0.027)$ (Table I).

\section{DISCUSSION}

The present study is one of several studies that assessed the improvements in LV diastolic and systolic function among patients with severe AS after AVR. The results (obtained using TDI) of the patients who underwent isolated AVR and those who underwent concomitant AVR with CABG were also compared in the present study. We demonstrated that TDI can be used to detect improvements in LV diastolic and systolic function in patients with severe AS after AVR. TDI has been shown to be useful in detecting subtle alterations in myocardial function ${ }^{(6)}$ and evaluating regional systolic LV contraction. ${ }^{(9-11)}$ The use of TDI may be better than conventional measurements which rely on endocardial movement and wall thickening. ${ }^{(9,10)}$ Furthermore, in conventional echocardiographic assessments, global LV diastolic function can only be indirectly derived from the parameters of LV filling. ${ }^{(11,12)}$ As TDI can provide measurements of myocardial 
Table II. Comparison of echocardiographic parameters of all patients $(n=23)$ before and after aortic valve replacement (AVR).

\begin{tabular}{|c|c|c|c|}
\hline \multirow[t]{2}{*}{ Parameter } & \multicolumn{2}{|c|}{ Mean \pm standard deviation } & \multirow[t]{2}{*}{ p-value } \\
\hline & Before AVR & After AVR & \\
\hline LV end-diastolic volume $(\mathrm{mL})$ & $110 \pm 29$ & $106 \pm 36$ & NS \\
\hline LV end-systolic volume (mL) & $42 \pm 23$ & $43 \pm 29$ & NS \\
\hline LV ejection fraction (\%) & $57 \pm 18$ & $60 \pm 9$ & NS \\
\hline LV mass index $\left(\mathrm{g} / \mathrm{m}^{2}\right)$ & $128 \pm 33$ & $116 \pm 34$ & NS \\
\hline Cardiac index (L/min. $\left.\mathrm{m}^{2}\right)$ & $3.05 \pm 0.99$ & $2.93 \pm 0.70$ & NS \\
\hline$E(\mathrm{~cm} / \mathrm{s})$ & $75 \pm 18$ & $85 \pm 26$ & NS \\
\hline$A(\mathrm{~cm} / \mathrm{s})$ & $88 \pm 23$ & $92 \pm 24$ & NS \\
\hline Mitral inflow deceleration time (ms) & $209 \pm 59$ & $209 \pm 76$ & NS \\
\hline Aortic transvalvular peak velocity $(\mathrm{cm} / \mathrm{s})$ & $416 \pm 92$ & $269 \pm 50$ & $<0.001$ \\
\hline Aortic transvalvular mean gradient $(\mathrm{mmHg})$ & $44 \pm 19$ & $17 \pm 7$ & $<0.001$ \\
\hline Aortic valve area $\left(\mathrm{cm}^{2}\right)$ & $0.88 \pm 0.40$ & $1.36 \pm 0.45$ & $<0.001$ \\
\hline Aortic valve resistance (dyne.s $/ \mathrm{cm}^{5}$ ) & $236 \pm 166$ & $101 \pm 56$ & 0.006 \\
\hline$E^{\prime}(\mathrm{cm} / \mathrm{s})$ & $8.11 \pm 2.45$ & $9.73 \pm 2.99$ & 0.011 \\
\hline$A^{\prime}(\mathrm{cm} / \mathrm{s})$ & $11.19 \pm 3.55$ & $13.07 \pm 3.24$ & 0.025 \\
\hline $\mathrm{S}^{\prime}(\mathrm{cm} / \mathrm{s})$ & $8.07 \pm 1.77$ & $10.09 \pm 2.85$ & 0.004 \\
\hline$E / E '$ & $10.10 \pm 4.33$ & $10.03 \pm 4.41$ & NS \\
\hline \multicolumn{4}{|l|}{ Isolated AVR patients $(n=13)$} \\
\hline$E^{\prime}(\mathrm{cm} / \mathrm{s})$ & $8.27 \pm 2.47$ & $10.56 \pm 2.57$ & 0.019 \\
\hline$A^{\prime}(\mathrm{cm} / \mathrm{s})$ & $12.02 \pm 3.62$ & $14.30 \pm 2.92$ & 0.035 \\
\hline $\mathrm{S}^{\prime}(\mathrm{cm} / \mathrm{s})$ & $8.79 \pm 1.63$ & $11.02 \pm 3.12$ & 0.038 \\
\hline \multicolumn{4}{|l|}{ CABG + AVR patients $(n=10)$} \\
\hline$E^{\prime}(\mathrm{cm} / \mathrm{s})$ & $7.69 \pm 2.47$ & $8.58 \pm 3.12$ & NS \\
\hline$A^{\prime}(\mathrm{cm} / \mathrm{s})$ & $9.54 \pm 2.49$ & $11.76 \pm 3.12$ & NS \\
\hline $\mathrm{S}^{\prime}(\mathrm{cm} / \mathrm{s})$ & $7.23 \pm 1.51$ & $8.88 \pm 1.77$ & 0.028 \\
\hline
\end{tabular}

A: late diastolic transmitral filling velocity; A': late diastolic mitral annular velocity; AVR: aortic valve replacement; CABG: coronary artery bypass grafting; E: early diastolic transmitral filling velocity; E': early diastolic mitral annular velocity; LV: left ventricular; NS: not significant; S': systolic mitral annular velocity
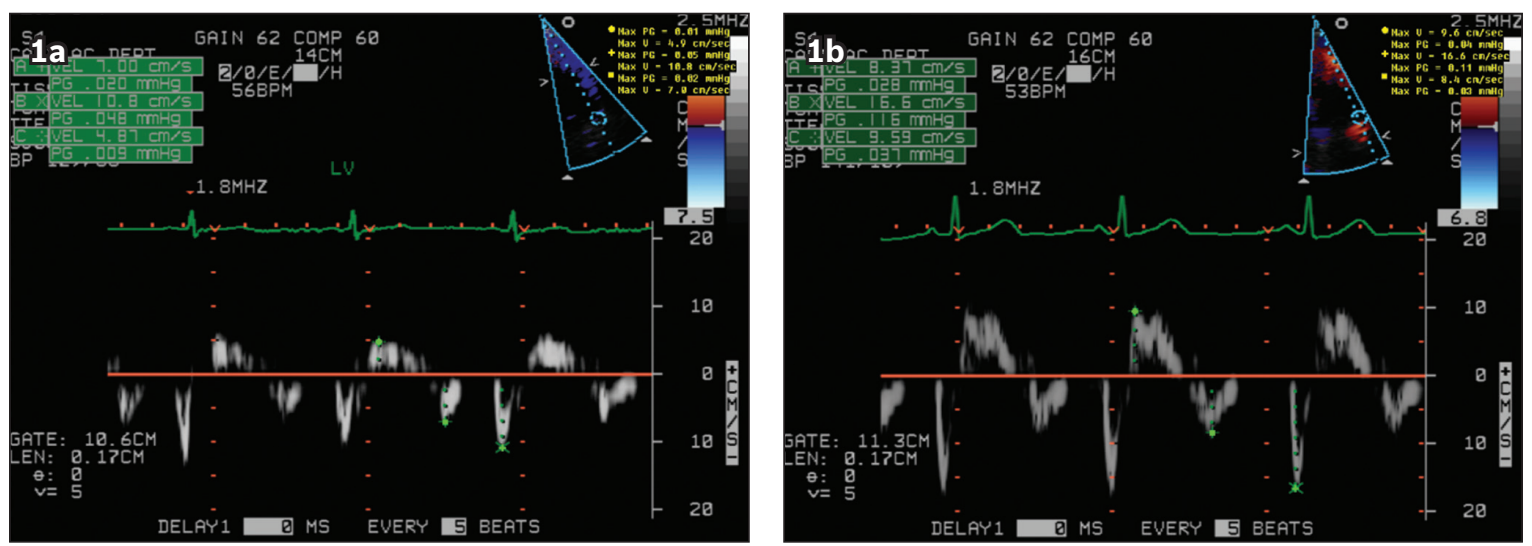

Fig. 1 Tissue Doppler images show the improvement in mitral annular velocities (a) before and (b) after aortic valve replacement.

tissue velocity, it allows for the quantitative assessment of both regional systolic and diastolic LV function. ${ }^{(13,14)}$ The motions caused by longitudinally directed fibres that are found mainly in the LV subendocardium and subepicardium can be assessed using myocardial systolic annular velocities obtained from TDI. ${ }^{(15)}$ These motions also contribute to global long-axis function. ${ }^{(16,17)}$ Diastolic annular velocities from TDI can also be used to assess LV diastolic function. In the present study, we showed that there was a significant increase in $\mathrm{E}^{\prime}, \mathrm{A}^{\prime}$ and $\mathrm{S}^{\prime}$ after $\mathrm{AVR}$, which is consistent with past literature demonstrating improvement in LV diastolic and systolic function after AVR in patients with severe AS. ${ }^{(18,19)}$
In severe AS, chronically increased afterload leads to progressive LV remodelling and myocardial hypertrophy. ${ }^{(20)}$ Despite this compensatory mechanism, which reduces LV endsystolic wall stress, increased metabolic demand, impaired LV relaxation and reduced LV compliance may occur in the long run. This results in the inability of the left ventricle to attain normal filling pressure during diastole. The increase in LV diastolic pressure leads to an increased dependence on atrial contribution to LV filling. There is progressive dilatation of the left atrium, resulting in progressive atrial dysfunction. ${ }^{(21,22)} A^{\prime}$ is a good predictor of clinical outcomes; a lower $\mathrm{A}^{\prime}$ suggests reduced atrial 
booster pump function. ${ }^{(21)}$ Similarly, increased left atrial volume is a reproducible surrogate marker of adverse cardiovascular outcomes. ${ }^{(23)}$ AVR reduces the pressure overload caused by severe AS, thus inducing a trend toward the normalisation of LV haemodynamics, with progressive remodelling of fibrotic and myocyte components. ${ }^{(24)}$

A previous study that compared patients who underwent isolated AVR and patients who underwent AVR with concomitant CABG showed that the former group had an excellent long-term prognosis, with significant improvement in LV diastolic and systolic function after AVR. ${ }^{(25)}$ This is consistent with the results of the present study; we observed significant improvements in $\mathrm{E}^{\prime}, \mathrm{A}^{\prime}$ and $\mathrm{S}^{\prime}$ among the patients who underwent isolated AVR. Among the patients who underwent concomitant AVR with CABG, the only significant improvement was in $S^{\prime}$. The baseline TDI parameters of the patients who underwent concomitant AVR with CABG were also lower than those of the patients who underwent isolated AVR. The former group of patients may have more comorbidities, and worsened LV systolic and diastolic functions in the presence of CAD, which confounded the outcomes. ${ }^{(26,27)}$ An alternative explanation would be that more time is needed for recovery of myocardial function in this group of patients. It is possible that since the $\mathrm{S}^{\prime}$ of this patient group has a significantly lower baseline level ( $p<0.01$ ) (as compared to those who underwent isolated AVR), it may have been the first parameter to improve after AVR. However, we did not conduct longitudinal follow-up on the patients and were not able to investigate this.

The present study was not without limitations. The sample size of the study was small. Secondly, the durations between the preoperative and postoperative echocardiographic examinations varied due to the retrospective nature of the study. Nonetheless, there was no significant difference between the patients who underwent isolated $A V R$ and those who underwent AVR with concomitant CABG (Table II). Patients with atrial fibrillation, pacing devices and concomitant significant mitral regurgitation were excluded. This is because transmitral A and mitral annular $\mathrm{A}^{\prime}$ are not available for analysis in patients with atrial fibrillation, TDI diastolic parameters may be affected by cardiac pacing, and patients with significant mitral regurgitation have increased transmitral E. TDI velocities may be reduced in patients with significant mitral annular calcification - a trait frequently associated with degenerative AS. ${ }^{(28)}$ It is known that abnormal septal motion may occur after cardiac surgery and may affect mitral annular diastolic velocities. ${ }^{[24]}$ However, all of the patients who were included in the study would have been similarly affected as they all underwent surgery. Another limitation is that pulse wave TDI of the mitral annulus may be affected by tethering and all the echocardiographic parameters measured may be sensitive to load. ${ }^{(29)}$ However, we ascertained that the echocardiographic examinations carried out in the present study, including TDI, occurred when the patients were haemodynamically stable and did not have tachycardia. Changes in TDI parameters could have been confounded by changes in LV preload and afterload, due to the cessation of medications (e.g. diuretics, vasodilators and nitrates) after AVR. However, TDI parameters are less affected by changes in loading conditions than conventional Doppler parameters. Lastly, newer myocardial deformation indices (e.g. global longitudinal strain by speckle tracking echocardiography) were not measured. These parameters are dependent on optimal LV endocardial border delineation and require detailed post-processing of images. On the other hand, TDI is easily performed and available in most echocardiographic laboratories.

To conclude, TDI is able to detect improvements in LV systolic and diastolic functions in patients with severe AS after AVR. There was less improvement in the TDI-derived diastolic parameters among patients who underwent concomitant AVR with CABG than among those who underwent isolated AVR.

\section{REFERENCES}

1. Rahimtoola SH. Valvular heart disease: a perspective on the asymptomatic patient with severe valvular aortic stenosis. Eur Heart J 2008; 29:1783-90.

2. Otto CM. Valvular aortic stenosis: disease severity and timing of intervention. J Am Coll Cardiol 2006; 47:2141-51.

3. American College of Cardiology/American Heart Association Task Force on Practice Guidelines; Society of Cardiovascular Anesthesiologists; Society for Cardiovascular Angiography and Interventions; Society of Thoracic Surgeons, Bonow RO, Carabello BA, et al. ACC/AHA 2006 guidelines for the management of patients with valvular heart disease: a report of the American College of Cardiology/American Heart Association Task Force on Practice Guidelines (writing committee to revise the 1998 Guidelines for the Management of Patients with Valvular Heart Disease): developed in collaboration with the Society of Cardiovascular Anesthesiologists: endorsed by the Society for Cardiovascular Angiography and Interventions and the Society of Thoracic Surgeons. Circulation 2006; 114:e84-231.

4. Vahanian A, Alfieri O, Andreotti F, et al; ESC Committee for Practice Guidelines (CPG); Joint Task Force on the Management of Valvular Heart Disease of the European Society of Cardiology (ESC); European Association for Cardio-Thoracic Surgery (EACTS). Guidelines on the management of valvular heart disease (version 2012): the Joint Task Force on the Management of Valvular Heart Disease of the European Society of Cardiology (ESC) and the European Association for Cardio-Thoracic Surgery (EACTS). Eur J Cardiothorac Surg 2012; 42:S1-44.

5. Stefanini GG, Stortecky S, Meier B, Windecker S, Wenaweser P. Severe aortic stenosis and coronary artery disease. Eurolntervention 2013; 9 Suppl: S63-8.

6. Yu CM, Sanderson JE, Marwick TH, Oh JK. Tissue Doppler imaging a new prognosticator for cardiovascular diseases. J Am Coll Cardiol 2007; 49:1903-14

7. Skjaerpe T, Hegrenaes L, Hatle L. Noninvasive estimation of valve area in patients with aortic stenosis by Doppler ultrasound and two-dimensional echocardiography. Circulation 1985; 72:810-8.

8. Baumgartner $\mathrm{H}$, Hung J, Bermejo J, et al; American Society of Echocardiography; European Association of Echocardiography. Echocardiographic assessment of valve stenosis: EAE/ASE recommendations for clinical practice. J Am Soc Echocardiogr 2009; 22:1-23.

9. Schiller NB, Shah PM, Crawford M, et al. Recommendations for quantitation of the left ventricle by two-dimensional echocardiography. American Society of Echocardiography Committee on Standards, Subcommittee on Quantitation of Two-Dimensional Echocardiograms. J Am Soc Echocardiogr 1989; 2:358-67.

10. Feneley MP, Hickie JB. Validity of echocardiographic determination of left ventricular systolic wall thickening. Circulation 1984; 70:226-32.

11. Sutherland GR, Steward MJ, Groundstroem KW, et al. Colour Doppler myocardial imaging: a new technique for the assessment of myocardial function. J Am Soc Echocardiogr 1994; 7:441-58

12. Fleming AD, Xia X, McDicken WN, Sutherland GR, Fenn L. Myocardial velocity gradient by Doppler imaging. Br J Radiol 1994; 67:679-88.

13. Donovan CL, Armstrong WF, Bach DS. Quantitative Doppler tissue imaging of the left ventricular myocardium: validation in normal subjects. Am Heart J 1995; 130:100-4.

14. Miyatake K, Yamagishi M, Tanaka N, et al. New method for evaluating left ventricular wall motion by color-coded tissue Doppler imaging: in vitro and in vivo studies. J Am Coll Cardiol 1995; 25:717-24. 
15. Yamazaki N, Mine $Y$, Sano A, et al. Analysis of ventricular wall motion using color-coded tissue Doppler imaging system. Jpn J Appl Phys 1994; 33:3141-6

16. Lancellotti P, Moonen M, Magne J, et al. Prognostic effect of long-axis left ventricular dysfunction and B-type natriuretic peptide levels in asymptomatic aortic stenosis. Am J Cardiol 2010; 105:383-8.

17. Bruch C, Stypmann J, Grude M, et al. Tissue Doppler imaging in patient with moderate to severe aortic valve stenosis: clinical usefulness and diagnostic accuracy. Am Heart J 2004; 148:696-702.

18. Krayenbuehl H, Hess OM, Monrad ES, et al. Left ventricular myocardia structure in aortic valve disease before, intermediate, and late after aortic valve replacement. Circulation 1989; 79:744-55.

19. Galema TW, Yap SC, Soliman OI, et al. Recovery of long-axis left ventricular function after aortic valve replacement in patients with severe aortic stenosis. Echocardiography 2010; 27:1177-81.

20. Lund O, Erlandsen M, Dørup I, et al. Predictable changes in left ventricular mass and function during ten years after valve replacement for aortic stenosis. J Heart Valve Dis 2004; 13:357-68.

21. Poh KK, Chan MY, Yang H, et al. Prognostication of valvular aortic stenosis using tissue Doppler echocardiography: underappreciated importance of late diastolic mitral annular velocity. J Am Soc Echocardiogr 2008; 21:475-81.

22. Dalsgaard M, Egstrup K, Wachtell K, et al. Left atrial volume in patients with asymptomatic aortic valve stenosis (the Simvastatin and Ezetimibe in Aortic Stenosis study). Am J Cardiol 2008; 101:1030-4.

23. Abhayaratna WP, Seward JB, Appleton $C P$, et al. Left atrial size: physiologic determinants and clinical applications. J Am Coll Cardiol 2006; 47:2357-63.

24. Giorgi D, Di Bello V, Talini E, et al. Myocardial function in severe aortic stenosis before and after aortic valve replacement: a Doppler tissue imaging study. J Am Soc Echocardiogr 2005; 18:8-14.

25. Kvidal P, Bergström R, Hörte LG, Ståhle E. Observed and relative survival after aortic valve replacement. I Am Coll Cardiol 2000; 35:747-56.

26. Peverill RE, Gelman JS, Mottram PM, et al. Factors associated with mitral annular systolic and diastolic velocities in healthy adults. J Am Soc Echocardiogr 2004; 17:1146-54.

27. Yu CM, Fung JW, Zhang Q, et al. Tissue Doppler echocardiographic evidence of atrial mechanical dysfunction in coronary artery disease. Int J Cardiol 2005; 105:178-85.

28. Muhammad N, Yap SE, Seet D, Chia BL, Poh KK. Does oral calcium intake or body habitus relate to the degree of valvular calcification and adverse events in patients with severe aortic stenosis? Int J Cardiol 2015; 180:74-5.

29. Abraham TP, Dimaano VL, Liang HY. Role of tissue Doppler and strain echocardiography in current clinical practice. Circulation 2007; 116:2597-609. 